SHORT REPORT

\title{
Medical and environmental risk factors for sporadic frontotemporal dementia: a retrospective case-control study
}

\author{
S M Rosso, E-J Landweer, M Houterman, L Donker Kaat, C M van Duijn, J C van Swieten
}

J Neurol Neurosurg Psychiatry 2003;74:1574-1576

A retrospective case-control study was carried out on 80 patients with sporadic frontotemporal dementia and 124 age, sex, and surrogate informant matched controls with respect to various medical and environmental risk factors. Head trauma was associated with an odds ratio of $3.3195 \%$ confidence interval (CI), 1.3 to 8.1). Although recall bias may play a role, the frontal lobes are known to be especially vulnerable to even mild head trauma. Thyroid disease was associated with a 2.5 times increased risk of frontotemporal dementia $(95 \% \mathrm{Cl}, 0.9$ to 7.9$)$, which was not statistically significant $(p=0.09)$ owing to limited power. As altered thyroid hormone status has been observed before in frontotemporal dementia, future studies will be important to confirm this observation.
$\mathrm{F}$ rontotemporal dementia is a neurodegenerative disorder with a predominantly presenile onset of behavioural changes and cognitive decline. ${ }^{1}$ Following the identification of tau gene mutations in familial forms of this disorder, there is increasing interest in genetic factors that may predispose to the disease. About $60 \%$ of patients with frontotemporal dementia have no family history of dementia and are considered to be sporadic cases. Genetic factors, such as apolipoprotein $\mathrm{E}$ genotype and the $\mathrm{Hl}$ haplotype of the tau gene, have been inconsistently associated with the sporadic form of the disease. ${ }^{2-4}$ To our knowledge, no studies have been reported to date addressing non-genetic risk factors for this condition, in contrast to other neurodegenerative disorders such as Alzheimer's disease, Parkinson's disease, and progressive supranuclear palsy. The identification of new risk factors may help understand the mechanisms underlying the aetiology of sporadic frontotemporal dementia. We therefore undertook a retrospective case-control study of 80 patients with this form of dementia and 124 age, sex, and surrogate informant matched controls with respect to possible medical and environmental risk factors.

\section{METHODS}

\section{Cases}

Cases were identified through our nationwide study on frontotemporal dementia, which attempted to ascertain all patients with this disorder in the Netherlands between January 1994 and January 2002. All neurological, gerontopsychiatric, and nursing home physicians received regular postal enquiries requesting referral of all suspected cases. ${ }^{5}$ The diagnosis was based on international clinical criteria, ${ }^{1}$ and included a progressive behavioural disorder with insidious onset; affective symptoms; speech disorder; frontal executive dysfunction with preserved spatial orientation on psychometric evaluation; and selective frontotemporal atrophy (on computed tomography or magnetic resonance imaging) or hypoperfusion (single photon emission com- puted tomography). Cases were considered to have a sporadic form of frontotemporal dementia if there were no first degree relatives with dementia before the age of 70 , nor any tau mutations identified.

\section{Controls}

For each case at least one but if possible two controls were identified through either the nursing home where the patient resided or the general practitioner. Collaborating physicians were asked to recruit persons willing to answer a verbatim questionnaire as a surrogate respondent regarding a family member (spouse, parent, or sibling) whose current age $( \pm 5$ years) and sex were matched to that of the patient. These family members then served as surrogate informant matched controls, although only the relative was interviewed. We excluded controls if they had ever consulted a physician with memory complaints or had been diagnosed with dementia.

\section{Data collection}

Because patients with frontotemporal dementia are unreliable as historians, the information was collected from a close relative of the patient, the surrogate informant. Only spouses, offspring, and siblings were questioned, as information from more distant relatives is unreliable. A structured verbal interview was administered without formal blinding by one of the investigators to the surrogate informant of both cases and controls.

A risk factor was considered positive only if it preceded the date of onset of frontotemporal dementia in the matched case. The investigators were blinded to the information available from the medical records of the patients with dementia to avoid information bias. Questions regarded history of the medical problems (table 1), the level of education (divided into three categories: primary school only; secondary school or lower/intermediate professional education; and higher professional education or university), employment or hobbies associated with exposure to chemicals, pesticides or insecticides, and smoking and alcohol consumption (table 2).

Head trauma was regarded as positive if it was followed by severe headache, nausea, blurred or double vision, vertigo, amnesia, or loss of consciousness. Severe head trauma was present only if the trauma was followed by loss of consciousness. Thyroid problems were positive if confirmed by a specialist or if thyroid related drugs were being prescribed by a general practitioner. We also collected information on the practice of sports associated with chronic head trauma (for example, boxing, football). Smoking was measured in pack-years-that is, the average number of cigarette packs smoked daily times the number of years the individual had smoked. Alcohol consumption was measured in drinks per day and considered mild to moderate if individuals had taken between one and three drinks a day, and severe if they had taken more than three drinks a day, for at least 10 years. 
Table 1 History of medical problems in cases and controls

\begin{tabular}{|c|c|c|c|}
\hline & $\begin{array}{l}\text { Sporadic FTD } \\
(n=80)\end{array}$ & $\begin{array}{l}\text { Controls } \\
(n=124)\end{array}$ & $\mathrm{p}$ Value \\
\hline Hypertension & 11 (14\%) & $18(15 \%)$ & 0.9 \\
\hline Diabetes mellitus & $5(6 \%)$ & $3(2 \%)$ & 0.2 \\
\hline High cholesterol & $6(8 \%)$ & $7(6 \%)$ & 0.6 \\
\hline $\begin{array}{l}\text { Myocardial } \\
\text { infarction }\end{array}$ & 0 & $3(2 \%)$ & 0.1 \\
\hline Stroke & 0 & 0 & - \\
\hline $\begin{array}{l}\text { Meningitis/ } \\
\text { encephalitis }\end{array}$ & 0 & 0 & - \\
\hline Seizures & 0 & $1(1 \%)$ & 0.4 \\
\hline $\begin{array}{l}\text { Head trauma } \\
\text { with loss of } \\
\text { consciousness }\end{array}$ & $\begin{array}{l}19(24 \%) \\
5(6 \%)\end{array}$ & $\begin{array}{l}10(8 \%) \\
3(2 \%)\end{array}$ & $\begin{array}{l}0.002 \\
0.2\end{array}$ \\
\hline Thyroid disease & 11 (14\%) & $6(5 \%)$ & 0.03 \\
\hline $\begin{array}{l}\text { Headache, > once } \\
\text { a month }\end{array}$ & $20(25 \%)$ & $25(20 \%)$ & 0.4 \\
\hline Migraine & $12(15 \%)$ & $9(7 \%)$ & 0.08 \\
\hline Herpes zoster & $4(5 \%)$ & $7(6 \%)$ & 0.8 \\
\hline Cold sores & $30(38 \%)$ & $32(26 \%)$ & 0.08 \\
\hline Severe influenza & $5(6 \%)$ & $9(7 \%)$ & 0.8 \\
\hline
\end{tabular}

Values are $\mathrm{n}(\%)$.

FTD, frontotemporal dementia.

\section{Statistical analysis}

Statistical procedures were undertaken using the Statistical Package of Social Sciences (SPSS) software. Differences between cases and controls were analysed using the $\chi^{2}$ test for categorical variables and the $t$ test for continuous data. Variables with a probability $(\mathrm{p})$ value of less than 0.05 were evaluated in a multivariate analysis in which these variables were studied simultaneously. Conditional logistic regression was used to determine both crude and adjusted odd ratios (OR) adjusted for age, sex, and surrogate respondent relationship, with each case and its control(s) classified as a stratum; 95\% confidence intervals (CI) were calculated.

\section{RESULTS}

We interviewed surrogate respondents of all 80 ascertained cases of frontotemporal dementia who fulfilled the criteria for the sporadic form. Nursing home physicians and general practitioners referred 124 possible control subjects to us, all of whom we interviewed. In a recent epidemiological survey of the prevalence of frontotemporal dementia in the Netherlands, we estimated that about $40 \%$ of the cases were not identified. ${ }^{6}$ Unfortunately we do not have any information on risk factors in patients not referred to us, nor on control subjects who did not give permission to the collaborating physicians to be interviewed by us.

The mean (SD) age of the 80 patients with frontotemporal dementia was 64.3 (9.4) years, and in the 124 controls, 62.5 (10.2) years $(p=0.2)$. The surrogate respondent was a spouse in 54 cases $(67 \%)$, an offspring in 22 cases $(28 \%)$, and a sibling in four cases $(5 \%)$. Regarding a history of medical problems (table 1), both head trauma and thyroid disease had a $\mathrm{p}$ value of less than 0.05 . With conditional logistic regression, only head trauma remained a significant independent risk factor for frontotemporal dementia and was associated with a crude OR of 3.6 (95\% CI, 1.5 to 8.8 ) and an adjusted OR of 3.3 (1.3 to 8.1). The time between head trauma and the onset of dementia spanned several decades in some patients. Head trauma followed by loss of consciousness was also more frequent in patients with frontotemporal dementia, but was too infrequent to be a significant risk factor. Thyroid disease was not associated with a significant adjusted OR (crude OR, 2.9 ( 1.1 to 8.0), adjusted OR, 2.5 (0.9 to 7.9)). The level of education in both cases and controls was similar (table 2). Cases did not practice contact sports
Table 2 Level of education, smoking, alcohol consumption, and exposure to toxins

\begin{tabular}{|c|c|c|c|}
\hline & $\begin{array}{l}\text { Sporadic FTD } \\
(\mathrm{n}=80)\end{array}$ & $\begin{array}{l}\text { Controls } \\
(n=124)\end{array}$ & p Value \\
\hline Level of education* & & & 0.9 \\
\hline Low & $17(21 \%)$ & $27(22 \%)$ & \\
\hline Intermediate & $47(59 \%)$ & $70(56 \%)$ & \\
\hline High & $16(20 \%)$ & 27 (22\%) & \\
\hline Smoking & & & 0.7 \\
\hline Never & $32(40 \%)$ & $53(43 \%)$ & \\
\hline$<20$ pack-years & $27(34 \%)$ & $45(36 \%)$ & \\
\hline$\geqslant 20$ pack-years & $21(26 \%)$ & $26(21 \%)$ & \\
\hline Alcohol consumption & & & 0.4 \\
\hline$<1$ drink/day & $28(35 \%)$ & $55(44 \%)$ & \\
\hline 1 to 3 drinks/day & $46(57 \%)$ & 60 (49\%) & \\
\hline$>3$ drinks/day & $6(8 \%)$ & $9(7 \%)$ & \\
\hline $\begin{array}{l}\text { Exposure to } \\
\text { chemicals, pesticides } \\
\text { or insecticidest }\end{array}$ & & & 0.08 \\
\hline Yes & $16(20 \%)$ & $14(11 \%)$ & \\
\hline No & $64(80 \%)$ & $110(89 \%)$ & \\
\hline
\end{tabular}

Values are $\mathrm{n}(\%)$

*Low, primary school or less; medium, secondary school, including professional or vocational training; high, higher professional education and university.

tIncluding: asbestos, ammonia, petroleum, acetone, paint, chloride, mercury, radioactivity, and so on.

FTD, frontotemporal dementia.

associated with chronic head trauma, nor were they exposed to chemicals, pesticides, or insecticides more regularly than control subjects. Smoking and alcohol consumption were not associated with higher or lower risks of frontotemporal dementia (table 2).

\section{DISCUSSION}

To our knowledge, this is the first case-control study on the medical and environmental risk factors in patients with sporadic frontotemporal dementia. Head trauma was associated with a 3.3-fold increase in risk of this condition, and thyroid disease with a 2.5-fold increase (though the latter was not statistically significant in adjusted conditional regression analysis). Neither smoking nor alcohol use was associated with this form of dementia, nor was exposure to chemicals, pesticides or insecticides.

Head trauma, a significant risk factor for frontotemporal dementia in the current study, has also been associated with Alzheimer's disease in several case-control studies, although this has not been confirmed in prospective studies. ${ }^{78} \mathrm{Head}$ trauma followed by loss of consciousness-a measure of severe brain injury-also appeared to be more frequent in patients with frontotemporal dementia, although this was not significant owing to the rarity of the occurrence. The frontal lobes are especially sensitive to head trauma, and experimental animal models and necropsy studies have shown axonal damage and dysfunction, mostly localised in the frontal lobes, following even mild traumatic brain injury. ${ }^{9}$ However, we cannot exclude recall bias as a factor in the observed association, as surrogate informants may be more prone to remember mild head trauma (followed only by subjective symptoms such as dizziness and headache) in patients than in controls, as a possible cause of their dementia. If this were a major factor, however, we would expect there to be more neurological disorders associated with an increased frequency of head trauma.

Thyroid disease was linked to a 2.5-fold increased risk of frontotemporal dementia, although the association was not significant after adjustment. It is well known that thyroid problems can lead to cognitive disturbance and even dementia, but thyroid disease may also be associated specifically with frontotemporal dementia. Thus, first, experi- 
mental studies have shown that splicing of juvenile and adult tau mRNA variants is regulated by thyroid hormone, ${ }^{10}$ and Pick's disease, one of the tauopathies, is a common cause of the clinical syndrome of frontotemporal dementia. Second, a study of thyroid hormone levels in different dementia syndromes showed that abnormalities were rather common in frontotemporal dementia (38\%). ${ }^{11}$ Thus thyroid problems may play a role in the aetiology of sporadic frontotemporal dementia, and further studies will be important to evaluate this association.

Smoking and alcohol consumption have also been extensively studied in different neurodegenerative disorders. Smoking has been found to be protective against Parkinson's disease ${ }^{12}$ and likely to increase the risk of Alzheimer's disease, ${ }^{13}$ but in the current study neither effect was found. Also in contrast to the current study, light to moderate alcohol consumption (one to three drinks a day) has been shown to have a protective effect in Alzheimer's disease $^{14}$ but not in Parkinson's disease. ${ }^{15}$ A possible explanation why these risk factors were not identified in the current study may be that the clinical syndrome of frontotemporal dementia is caused by various different neuropathological substrates, and contrasting effects of risk factors may be diluted in a mixed group of patients. It will be important to conduct similar retrospective studies in patients with pathological verification of the disease in the future.

\section{ACKNOWLEDGEMENTS}

We thank all of the neurologists, nursing home physicians, and general practitioners who participated in collection of cases and controls for this study. This work was supported by a grant from the Netherlands Organisation for Scientific Research (NWO: project 94038-005).

\footnotetext{
Authors' affiliations

S M Rosso, E-J Landweer, M Houterman, L Donker Kaat, J C van

Swieten, Departments of Neurology, Erasmus Medical Centre

Rotterdam, Rotterdam, Netherlands

C M van Duijn, Departments of Epidemiology and Biostatistics, Erasmus Medical Centre Rotterdam

Competing interests: none declared
}

Correspondence to: Dr S M Rosso, Department of Neurology, Erasmus Medical Centre, Dr Molewaterplein 40, 3015 GD Rotterdam,

Netherlands; s.rosso@erasmusmc.nl

Received 18 December 2002

In revised form 4 May 2003

Accepted 6 May 2003

\section{REFERENCES}

1 Neary D, Snowden JS, Gustafson L, et al. Frontotemporal lobar degeneration: a consensus on clinical diagnostic criteria. Neurology 1998;51:1546-54.

2 Verpillat $\mathbf{P}$, Camuzat $A$, Hannequin D, et al. Association between the extended tau haplotype and frontotemporal dementia. Arch Neurol 2002;59:935-9.

3 Verpillat $\mathbf{P}$, Camuzat A, Hannequin D, et al. Apolipoprotein E gene in frontotemporal dementia: an association study and meta-analysis. Eur J Hum Genet 2002; 10:399-405.

4 Fabre SF, Forsell C, Viitanen M, et al. Clinic-based cases with frontotemporal dementia show increased cerebrospinal fluid tau and high apolipoprotein $\mathrm{E}$ epsilon4 frequency, but no tau gene mutations. Exp Neurol 2001;168:413-18.

5 Stevens $M$, van DC, Kamphorst W, et al. Familial aggregation in frontotemporal dementia. Neurology 1998;50:1541-5.

6 Rosso SM, Donker Kaat L, Baks T, et al. Frontotemporal dementia in the Netherlands; patient characteristics and prevalence estimates from a population-based study. Brain 2003;126:2016-22.

7 van Duijn CM, Tanja TA, Haaxma R, et al. Head trauma and the risk of Alzheimer's disease. Am J Epidemiol 1992;135:775-82.

8 Mehta KM, Ott A, Kalmijn S, et al. Head trauma and risk of dementia and Alzheimer's disease: the Rotterdam Study. Neurology 1999;53:1959-62.

9 De KJ, Twijnstra A, Leffers P. Diagnostic criteria and differential diagnosis of mild traumatic brain injury. Brain Inj 2001;15:99-106

10 Aniello F, Couchie D, Bridoux AM, et al. Splicing of juvenile and adult tau mRNA variants is regulated by thyroid hormone. Proc Natl Acad Sci USA 1991;88:4035-9.

11 Faldt R, Passant U, Nilsson K, et al. Prevalence of thyroid hormone abnormalities in elderly patients with symptoms of organic brain disease. Aging (Milano) 1996;8:347-53.

12 Morens DM, Grandinetti A, Reed D, et al. Cigarette smoking and protection from Parkinson's disease: false association or etiologic clue? Neurology 1995;45:1041-51

13 Ott A, Slooter AJ, Hofman A, et al. Smoking and risk of dementia and Alzheimer's disease in a population-based cohort study: the Rotterdam Study. Lancet 1998;351:1840-3.

14 Ruitenberg A, van SJ, Witteman JC, et al. Alcohol consumption and risk of dementia: the Rotterdam Study. Lancet 2002;359:281-6.

15 Checkoway H, Powers K, Smith-Weller T, et al. Parkinson's disease risks associated with cigarette smoking, alcohol consumption, and caffeine intake. Am J Epidemiol 2002;155:732-8. 